\title{
Sistema de gestión de la logística inversa en el sector de la refrigeración en la ciudad de Bogotá
}

Gerardo Avendaño Prieto* José Martín Díaz Pulido**

Fecha de recepción: 13 de septiembre de 2013

Fecha de aprobación: 20 de octubre de 2013

Pp. 215 a 241.

\section{RESUMEN}

La logística inversa en Colombia es poco conocida y aunque en ella se maneje el retorno de productos $\mathrm{y} / \mathrm{o}$ materiales, no se hace porque se tenga un conocimiento sobre el tema, no si porque existen algunas implementaciones aisladas en la recuperación de materiales y productos como lo son el reciclaje, la reutilización y la remanufactura (Monroy, Ahumada, y Cl, 2006). Las empresas deben tomarse su tiempo para entender las ventajas de la logística inversa con el propósito de: reducir problemas relacionados con las devoluciones; anticipar el cumplimiento de regulaciones y políticas de cuidado al ambiente; responder a las demandas actuales del mercado en cuanto a la responsabilidad ambiental sin incurrir en costos (Gaytan, 2012). Los consumidores están cada vez más conscientes de la importancia de adquirir productos sensibles al medio ambiente si mencionar las regulaciones impuestas por el gobierno que obligan a las organizaciones a replantear sus diseños, procesos y recuperación de sus productos y a colocarlos en el mercado.

\section{Palabras Clave}

Logística inversa, refrigeración, residuos, cadena de abastecimiento, reciclaje, refabricación, reutilización.

* Especialista en Ingeniería de Producción, Universidad Distrital Frencisco José de Caldas

** Ingeniería Industrial, Universidad Antonio Nariño 


\section{System of Reverse Logistics Management in the refrigeration sector of Bogota city}

\section{Abstract}

The Reverse Logistics used in Colombia is poorly understood; even though it handles the return of products and / or materials, it is not based on Reverse Logistics knowledge, but on some isolated implementations for the recovery of materials and products such as recycling, reusing and remanufacturing. It takes time for companies to understand the benefits of Reverse Logistics in order to: a) reduce problems with returns; b) anticipate regulatory compliance and environmental care policies; c) reply to current market demands in terms of environmental responsibility without incurring costs. Consumers are increasingly aware of the importance of acquiring environmentally sensitive products without mentioning governmentimposed regulations that companies require to rethink designs, processes and recovery of their products and their relocation in the market.

\section{KEY WORDS}

Reverse Logistics, Refrigeration, Waste, Supply Chain, Recycling. 


\section{Système de gestion de logistique inversée du secteur frigorifique de la ville de BOGOTA}

\section{RÉSUMÉ}

La logistique inversée, qui gère le retour des produits et matériaux vers leurs lieux de fabrication, est peu connue en Colombie. Cela est principalement dû au fait qu'il existe certaines structures isolées ou processus indépendants de récupération des produits et matériaux que sont le recyclage ou la réutilisation. Les entreprises mettent du temps à comprendre les avantages de la logistique inversée en terme de: a) réduction des problèmes liés aux retours-produits, b) anticipation de la conformité aux règlements et aux politiques de respect de l'environnement, c) réponse aux exigences actuelles du marché en matière de responsabilité environnementale.

Les consommateurs sont de plus en plus conscients de l'importance d'acquérir des produits respectant l'environnement sans omettre de mentionner les règlements imposés par les gouvernements obligeant les entreprises à repenser la conception des processus et la valorisation des produits mis sur le marché.

\section{Mots-clés}

Logistique Inversée, Déchets, Refroidissement, Chaîne d'Approvisionnement, Recyclage, Réutilisation. 


\section{Sistema de gestão da logística inversa no setor da refrigeração na cidade de Bogotá}

\section{ReSUMO}

A logística inversa é pouco conhecida na Colômbia, embora nela aconteça o retorno de produtos e/ou materiais, não é feita por ter conhecimento da logística inversa mas porque existem algumas implementações isoladas na recuperação de materiais e produtos como a reciclagem, a reutilização e a re-manufatura.

As empresas devem tomar tempo para entender as vantagens da logística inversa com o propósito de: a) Reduzir problemas relacionados às devoluções, b) Antecipar o cumprimento de regulações e políticas de cuidado do ambiente, c) Responder às demandas atuais do mercado com respeito à responsabilidade ambiental sem acrescentar os custos.

Os consumidores estão cada vez mais conscientes da importância de adquirir produtos sensíveis ao médio ambiente sem mencionar as regulações impostas pelo governo que obrigam às organizações a mudar seus desenhos, processos e recuperação de seus produtos e colocá-los no mercado.

\section{PalaVRas-ChaVe}

Logística Inversa, Refrigeração, Resíduos, Cadeia de Abastecimento, Reciclagem, Reutilização. 


\section{Introducción}

E n el año 1972 en Estocolmo, Suecia (Sánchez, 2002), se realizó la primera reunión de carácter mundial sobre medio ambiente, la Conferencia de las Naciones sobre el medio ambiente humano, en donde se hizo énfasis en los aspectos técnicos de la contaminación provocada por la industrialización acelerada, por la explosión demográfica y por la intensificación del proceso de crecimiento urbano. En este sentido su mayor logro fue crear una conciencia mundial sobre el deterioro del medio ambiente y abrir un debate sobre sus causas y consecuencias; de este modo, al implementar estrategias dieron mayor crecimiento a las organizaciones, entre las que se encuentra la logística inversa que promueve la prosperidad en la parte legal, social y económica.

En 1992 en Rio, Brasil, (Sánchez, 2002) se celebró la Conferencia de las Naciones Unidas sobre el medio ambiente y el desarrollo, o Cumbre de la Tierra, que marcó avances importantes en la comprensión de la relación desarrollo y medio ambiente. Se intentó definir el rumbo ambiental de la tierra para el final del siglo pasado y el inicio del presente y elaborar un documento representativo que preserve el sistema ecológico y asegure un desarrollo sostenible sin irrespetar los intereses de los distintos pueblos.

Se habló que para lograr el objetivo del desarrollo sostenible, se debe superar la pobreza absoluta en que vive más de la mitad de los habitantes de la tierra, modificar los modelos de desarrollo de los países industrializados y modificar los estilos de vida imperantes en los países desarrollados.

En Colombia (Sánchez, 2002), a partir de los años 90, se introdujo por primera vez una política ambiental sistemática y rigurosa que incluyó una sólida base institucional, técnica y financiera. En este período, los avances estuvieron marcados por el plan de desarrollo, básicamente a través de la Constitución de 1991. Se podría destacar de esta, los siguientes ejemplos: 
- Estableció la función ecológica de la propiedad.

- Señaló los deberes ambientales del Estado, los derechos ambientales de los ciudadanos.

- Ordenó la formulación de políticas ambientales como parte del Plan Nacional de Desarrollo.

- Introdujo la noción de desarrollo sostenible como meta para la sociedad.

Entre otros aspectos y la Ley 99 de 1993, estableció el Sistema Nacional Ambiental (SINA) que modificó la legislación en materia de licencias ambientales, tasas retributivas, tasas por uso del agua, destinación de recursos financieros para la gestión ambiental, y sanciones por infracción de las normas ambientales.

Desde el año 1990, en Colombia se inició con el término de globalización, la prevención y el control de la contaminación ambiental, al identificar los principales contribuyentes a este problema. Muestra del trabajo realizado, es el avance en la normatividad para prevenir la contaminación del aire y de otros recursos ambientales (Sánchez, 2002). Por lo tanto, se establece elaborar un documento que dé a conocer una propuesta desde el punto de vista de la ingeniería industrial, en la recuperación adecuada de los equipos de refrigeración, a través del planteamiento de un sistema de gestión de logística inversa en el sector de la refrigeración en la ciudad de Bogotá.

En este sentido, la presente investigación tiene como objetivo realizar una revisión de la literatura publicada en los últimos 11 años, sobre logística inversa a nivel de nacional e internacional, así como en la industria y el sector de la refrigeración, con el fin de identificar los avances de la logística inversa y los elementos claves que se deben tener en cuenta en la planeación, implementación y control de la misma.

En el primer momento de la investigación, se realizará una revisión de las definiciones de logística inversa, luego se hará una clasificación general de la literatura encontrada desde el año 2002, hasta lo que va corrido del año 2013, con el fin de caracterizarla y de este modo enfocarnos en los artículos que aportan conocimiento teórico a la temática e identificar los principios, sistemas de gestión y actividades correspondientes al tema de investigación. 


\section{Definición de logística inversa}

E xisten múltiples definiciones del concepto de logística inversa o la logística de la recuperación y el reciclaje. Desde el punto de vista ambiental, podría definirse como el conjunto de actividades logísticas de recogida, desmontaje y procesado de productos usados, partes de productos o materiales con vistas a maximizar el aprovechamiento de su valor y en general, su uso sostenible (Angulo, 2003).

Otra definición más técnica desde el Consejo Ejecutivo de Logística Inversa de Estados Unidos, el grupo PILOT y el grupo REVLOG de Europa, dice que la logística inversa es el proceso de planificación, implantación y control eficiente del flujo efectivo de costes y almacenaje de materiales, inventarios en curso y productos terminados, así como de la información relacionada, desde el punto de consumo al punto de origen, con el fin de recuperar valor o asegurar su correcta eliminación (Rogers y Tibben-Lembke, 1999).

Hawks (2006), define la logística mediante el Consejo de Gestión de la Logística como: "El proceso de planificación, implementación y control del flujo eficiente y rentable de las materias primas, en proceso de inventario de productos terminados e información relacionada desde el punto de origen hasta el punto de consumo con el fin de ajustarse a los requisitos del cliente". Logística inversa incluye todas las actividades que se mencionan en la definición anterior. La diferencia, es que esta comprende todas estas actividades, ya que operan en sentido inverso.

Partiendo de las definiciones anteriores, se puede decir que la logística inversa es el proceso de planificar, implementar y controlar el flujo eficiente y rentable de las materias primas, el inventario en proceso, productos terminados e información relacionada desde el punto de consumo hasta el punto de origen, con el fin de recuperar valor o su eliminación adecuada. Más precisamente, la logística inversa es el proceso de traslado de mercancías desde su lugar de destino final típico, con el propósito de captación de valor, o la correcta eliminación. 
Actividades de refabricación y renovación también pueden ser incluidos en la definición de la logística inversa. Hawks (2006) y otros autores como Carter y Ellram (1998); Knemeyer, Ponzurick, y Logar (2002); Brito y Daker (2003); Krikke, Blanc, y Velde (2003); Ortega (2003); Ravi y Shankar (2004); Fernández y Gómez (2005); Rubio (2007) y Council (2007), quienes definen la logística inversa como "El proceso de planificación, ejecución y control eficiente y rentable del flujo de materias primas, inventario en proceso, productos terminados e información relacionada desde el punto de consumo hasta el punto de origen con el fin de recuperar valor o realizar una correcta eliminación".

La logística inversa tiene diversas teorías dadas por distintos autores quienes: la definen así: "El proceso de mover bienes desde su destino final con el propósito de capturar valor, o una disposición final apropiada de los mismos" (Rogers y Tibben-Lembke, 1999).

Carter y Ellram (1998), indican que la logística inversa "es un proceso a través del cual las empresas pueden llegar a ser más eficientes medioambientalmente por medio del reciclaje, reutilización y reducción de la cantidad del material que utilizan".

Guide y V. D. R (2000), definen la logística inversa como, "la tarea de recuperar productos desechados; esto puede incluir embalajes y materiales de envío, y el acarreo regresivo de ellos hacia un punto central de recolección para su reciclado o remanufacturado".

La Red Española de Logística Inversa y el Consejo Ejecutivo de Logística Inversa, la definen como: "El proceso de planear, implementar y controlar efectiva y eficientemente el flujo de materias primas, inventario de productos en proceso, productos terminados e información relacionada desde el punto de consumo hacia el punto de origen, con el propósito de recapturar valor o una disposición apropiada".

Rojas, Salazar, Sepúlveda, et al (2006) y Bereciartúa y Echazarra (2007), sostienen que "por medio de la logística inversa se atiende la recuperación y reciclaje de envases, embalajes y residuos peligrosos, así como los procesos de retorno de excesos de inventario, devoluciones de clientes, productos obsoletos e inventarios estacionales". 
Monroy, Ahumada, (2006), definen la logística reversa como: "la gestión, manera eficiente y costo efectiva, del flujo de materiales, inventarios en proceso, productos terminados e información relacionada, destinados al reprocesamiento, reciclaje, reutilización o disposición final, desde el eslabón donde perdieron o disminuyeron su vida útil, para recuperar total o parcialmente su valor, disminuyendo el impacto medioambiental y los costos asociados".

La Asociación de Logística (ALV, 2008), dentro de su glosario, la define como: "Proceso de mover bienes y mercancías desde su punto final para recuperar parte de su valor o eliminarlos adecuadamente". Mediante este proceso, se recuperan y reciclan envases, embalajes, residuos peligrosos, retornos de excesos de inventario, devoluciones de clientes, productos obsoletos e inventarios estacionales. De igual forma, este proceso se adelanta al fin de la vida del producto y se ocupa de darle salida en mercados con mayor rotación.

En la Universidad Politécnica de Catalunya (Barcelona), en el trabajo de impacto de la logística inversa (2010), se habla del papel de esta y de la logística en el reciclado y gestión de materiales, por lo que desde una perspectiva más amplia incluye todo lo relacionado con las actividades encaminadas a reducción de material, reciclado, substitución y reutilización de materiales y residuos.

Desde la Universidad Nacional de Piura (2012), en su trabajo "Proceso modelos y casos, menciona que la logística inversa también es considerada como sinónimo de reutilización; aquí es donde se incluyen los productos reciclables; este proceso logístico brinda tres ventajas significativas básicas que pueden enumerarse en beneficios económicos, marco legal y conciencia social.

Logística inversa, oportunidad de negocio (2013), dentro de sus definiciones la contempla como uno de los servicios con mayor auge en los próximos años, debido a los beneficios que representa para las empresas, como la recuperación de mercancías para el cambio de empaques y su venta en otros puntos comerciales ya que incluye operaciones muy diversas como la gestión de material sobrante de inventario (Surplus Stocks), la devolución de compras a proveedores, la recuperación de embalajes y 
envases, la devolución de productos de electrodomésticos, electrónica e informática (los denominados gama blanca, gama marrón y gama gris) o en ocasiones, la gestión de residuos.

\section{Proceso de investigación}

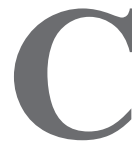
on base en la revisión previa de la definición de logística inversa, se tomaron las decisiones con respecto al periodo de tiempo sobre la indagación de artículos y palabras clave usadas como motor en el proceso de la investigación.

La revisión bibliográfica se realizó en las siguientes bases de datos: ISIs Web of Science ${ }^{\circledR}$, Science Direct ${ }^{\circledR}$, Taylor \& Francis $^{\circledR}$, Emerald $^{\circledR}, I E^{\circledR}$, Springerlink ${ }^{\circledR}$ y en el buscador Google Scholar, donde se encontraron artículos de divulgación y documentos oficiales. Se buscaron artículos publicados en el periodo comprendido entre el año 2002 hasta el año 2013 y las palabras claves utilizadas fueron: Reverse Logistics, Refrigeration ,Recovery, Waste, Supply Chain, Recycling, Remanufacturing, Reuse, Management System, Supply Chain y se filtraron solo los artículos que tuvieran que ver con ingeniería y ciencias de administrativas, dejando por fuera los que tuvieran que ver con medicina, meteorología y psicología que no tuvieran el enfoque logístico y administrativo. 


\section{Clasificación de artículos de logística inversa}

\subsection{Clasificación de tipo descriptivo}

Se realizaron primero clasificaciones de tipo descriptivo: año de publicación, revista de publicación y tipo de artículo (científico, divulgación, artículo en conferencia, resultado de proyecto de pregrado, resultado de proyecto de maestría y documento de trabajo) y según su aporte al conocimiento (teórico, modelo, caso de estudio).

Se revisaron 106 artículos, de los cuales 66 fueron clasificados como científicos, 12 de divulgación, 16 en conferencia, cuatro proyectos de grado en pregrado, dos proyecto de grado en maestría, una disertación doctoral y cuatro documentos de trabajo.

De los 106 artículos y documentos, el 54,82\% (48 artículos) fueron publicados en las revistas (Tabla 1), los demás fueron publicados cada uno en una revista diferente, teniendo la mayor cantidad de publicaciones la IEEE con 13 artículos, revista de conferencia internacional, y la revista Transportation Research Part E. con seis artículos

En cuanto al aporte al conocimiento de la logística inversa, se tomó como referencia de clasificación, la realizadapor Altay \& Green III, (OR/MS research in disaster operations management, 2006) con algunas modificaciones teniendo en cuenta el objetivo de esta revisión. Se clasificaron en teóricos aquellos artículos que investigan los principios y el desarrollo del sistema de la logística inversa, prueban alguna hipótesis, o proporcionan un marco sobre los problemas, desafíos y avances en el tema, también se incluyeron allí los artículos que tratan sobre el desarrollo de indicadores de desempeño del sistema. Aquellos artículos que desarrollan algún modelo o aplicación de modelo, se clasifican en otro grupo y a diferencia de Altay \& Green III no se clasificarán en desarrollo de aplicaciones, sino en casos a aquellos 
artículos que estudian cómo fue la atención de desastres particulares o que estudian casos específicos de atención (Tabla 2).

Tabla 1. Número de artículos científicos publicados por revista desde año 2002 hasta año 2013

\begin{tabular}{|l|c|}
\hline \multicolumn{1}{|c|}{ NOMBRE DE LA REVISTA } & CANTIDAD DE ARTíCULOS \\
\hline IEEE & 13 \\
\hline Transportation Research Part E & 6 \\
\hline $\begin{array}{l}\text { Supply Chain Management: An International } \\
\text { Journal }\end{array}$ & 6 \\
\hline $\begin{array}{l}\text { International Journal of Physical Distribution } \\
\text { \& Logistics Management }\end{array}$ & 5 \\
\hline Computers \& Operations Research & 4 \\
\hline Management Research News & 2 \\
\hline Journal of the Operational Research Society & 2 \\
\hline $\begin{array}{l}\text { International Journal of Logistics Research and } \\
\text { Applications }\end{array}$ & 2 \\
\hline International Journal of Production Economics & 1 \\
\hline European Journal of Operational Research & 1 \\
\hline $\begin{array}{l}\text { International Journal of Productivity and } \\
\text { Performance Management }\end{array}$ & 1 \\
\hline Resources Conservation and Recycling & 1 \\
\hline Innovation & 1 \\
\hline Journal Computers and Industrial Engineering & $\mathbf{4 8}$ \\
\hline Total & \\
\hline
\end{tabular}

Fuente. Elaboración propia del autor.

Tabla 2. Número de artículos según aporte al conocimiento

\begin{tabular}{|l|c|c|}
\hline Aporte al conocimiento & $\begin{array}{c}\text { Cantidad de } \\
\text { artículos }\end{array}$ & Porcentaje \\
\hline Caso & 10 & $7,05 \%$ \\
\hline Modelo & 18 & $14,11 \%$ \\
\hline Teórico & 72 & $78.82 \%$ \\
\hline Total & 106 & $100 \%$ \\
\hline
\end{tabular}

Fuente. Elaboración propia del autor. 
Se puede observar que las publicaciones dedicadas a la discusión de los principios, prácticas, características, problemas, desafíos, avances y soluciones alrededor de la logística inversa, superan a las publicaciones dedicadas al desarrollo o aplicación de modelos matemáticos en torno a la gestión y operación de las actividades para atención de la industria.

En cuanto al número de publicaciones alrededor de la logística inversa, se observa que estas han venido en aumento desde el año 2002, observándose un incremento en los años 2012 con 20 publicaciones y 2013 con 14 .

Tabla No 3. Cantidad de publicaciones por año

\begin{tabular}{|c|c|}
\hline Año & $\begin{array}{c}\text { Cantidad de } \\
\text { publicaciones }\end{array}$ \\
\hline 2002 & 2 \\
\hline 2003 & 2 \\
\hline 2004 & 4 \\
\hline 2005 & 7 \\
\hline 2006 & 11 \\
\hline 2007 & 8 \\
\hline 2008 & 12 \\
\hline 2009 & 12 \\
\hline 2010 & 9 \\
\hline 2011 & 5 \\
\hline 2012 & 20 \\
\hline 2013 & 14 \\
\hline Total & 106 \\
\hline
\end{tabular}

Fuente. Elaboración propia del autor. 


\subsection{Clasificación de artículos teóricos}

Teniendo en cuenta que el objetivo de esta revisión es identificar estrategias de logística inversa a través de los avances y los elementos claves que se deben tener en cuenta en la planeación, implementación y control de la logística inversa, de los artículos previamente clasificados, se tomaron para la revisión de estrategias los 72 artículos clasificados como teóricos.

\subsection{Clasificación según etapas de procedimientos}

La literatura sobre logística inversa, contiene una serie de etapas en el procedimiento para gestionar de forma eficiente los productos introducidos por diferentes motivos en la cadena y recuperar el máximo de su valor. Para conceptualizar un sistema aplicado a la logística inversa, se tiene en cuenta el criterio de diferentes autores que permita generar un procedimiento lo más general posible, y que a su vez darán pautas para cada una de las etapas, estas son:

- Diagnóstico: el objetivo fundamental de esta etapa es realizar un diagnóstico medioambiental de la situación actual de la entidad que es objeto de estudio a través de una inspección rigurosa; se analizan las pérdidas, residuos, desechos, las entradas al sistema y toda la información que lleva consigo dicha fase.

- Fuentes de generación: se realiza un análisis de las fuentes de emisión de los volúmenes que se generan, de los clientes potenciales y de cuáles son todas las alternativas a analizar para pasar a la etapa siguiente. Es un tiempo de recopilación de información a través de registros fundamentalmente y evalúa qué impacto genera dicha fuente al medio ambiente para determinar forma de almacenamiento y recepción.

- Clasificación de residuos: en esta etapa, se evalúa el residuo teniendo en cuenta diferentes criterios como son: 
Estado del residuo o desecho.

Grado de peligrosidad.

Destino del residuo o desecho.

De acuerdo al origen.

" Grado de control que se tiene sobre el residuo o desecho.

» Caracterización del residuo o desecho.

»Almacenamiento temporal según su clasificación.

- Identificación de la estrategia a seguir: se determina cuál es la estrategia en cuanto al tratamiento que debe seguir el residuo o desecho, es decir, con un grupo de especialistas y consultando todas las normativas existentes que rigen en el país y a nivel internacional

- Determinación del tratamiento o destino: una vez identificada la estrategia a seguir, se realiza el tratamiento decidido o se pasa al destino final. A continuación se ofrece una pequeña descripción de los procesos de disposición y tratamiento más habituales:

» Vertido sin control: consiste en la acumulación de residuos sin ningún tipo de tratamiento.

» Vertido controlado o relleno sanitario: consiste en el almacenamiento de residuos en terrenos amplios que se excavan y se rellenan con capas alternativas de basura y de tierra compactadas.

» Incineración: proceso de combustión controlada a altas temperaturas, que transforma la fracción orgánica de los residuos en materiales inertes (cenizas) y gases.

» Reciclado: consiste en aprovechar los materiales de los que están hechos los residuos como materia prima para otras aplicaciones.

- Transporte y almacenamiento: las operaciones de recogida y transporte de los residuos representan entre el $60 \%$ y el $80 \%$ de los costos globales, y tiene en consecuencia, una gran importancia económica. En estas operaciones confluyen un conjunto de parámetros como la frecuencia de la recogida, los horarios de la misma, los equipos y el personal de recogida. 
- Medición y control: esta última etapa es de gran importancia ya que permite tener un control riguroso en cada una de las etapas, evaluar las mismas a través de indicadores y plantear diferentes alternativas de solución en cada momento. Una propuesta de indicadores, es:

»Cantidad de pérdidas o residuos.

»Costo total de gestión.

» Costo por pérdidas y residuos.

»Frecuencia de generación.

»Cumplimiento de la frecuencia de recogidas.

»Estructura de la composición de los residuos.

» Costo de almacenamiento y transportación.

» Comparación de los volúmenes potenciales a recuperarse en comparación con lo que realmente se recupera.

Además, es necesario tener el control a través de registros de una serie de informaciones, entre las cuales se encuentran:

- Llevar el control de la cantidad de pérdidas y residuos en (\%) y en volumen.

- Determinar el intervalo de tiempo entre la verificación y el control del volumen de residuos y pérdidas.

- Llevar el control de la clasificación, tratamiento y destino de los residuos y las pérdidas.

- Llevar el control de toda la información relacionada con el proceso.

A continuación se realiza la clasificación de los artículos teóricos según la fase que tratan (Tabla 4) y el criterio de diferentes, autores que permita generar un procedimiento lo más general posible, que a su vez, de pautas para cada una de las etapas (Tabla 5). 


\section{Tabla 4. Clasificación de los artículos teóricos}

\section{Artículos teóricos}

Acevedo, J. A., Ana J. Urquiaga Rodríguez, M. I. (2001). Gestión de la Cadena de Suministro. La Habana: Ed. ISPJAE.

Carrefour. (2003). La implantación de la Logística Inversa en una Multinacional de la Distribución. p.3.

García, A. A. (2004). Recomendaciones táctico-operativas para Implementar un programa de Logística Inversa. Eumed. Net.México.

Gómez, D. (1998). Evaluación de impacto ambiental. Madrid: Editorial Agrícola Española, S. A., pp.63-100.

Knudsen, J. A. ( 2005). Diseño y gestión de la cadena de suministro de los residuos agroindustriales de la caña de azúcar. Resumen de tesis de doctorado. Santa Clara, Ciudad de la Habana.

Matos. (1998). Modelo para el mejoramiento o diseño de un sistema de reciclaje para envases y embalajes. Resumen de tesis de Doctorado. Matanzas, Cuba.

REVLOG. (2002). GAT is reverse logistics?. The European Working Group Reverse Logistics.

Rogers \& Tibben-Lembke. (2003). RLEC. Reverse Logistics Executives' Council. p.9.

Pérez, A. et Al. (2003). Logística Inversa. Un nuevo reto en agestión de la cadena de suministro y un avance hacia el desarrollo sostenible. Ed. CIIL.

Cure, L. y Meza J. C. (2006). Logística Inversa: una herramienta de apoyo a la competitividad de las organizaciones. Recuperado de: http://ciruelo.uninorte. edu.co/pdf/ingenieria_desarrollo/20/logistica_inversa.pdf

García, A. A. (2009). Recomendaciones táctico-operativas para implementar un programa de logística Inversa. Bogotá.

Cure, L. (2006).Logística Inversa: una herramienta de apoyo a la competitividad de las organizaciones - Bogotá

Lacoba, S. (s.f.) El Sistema De Logística Inversa En La Empresa: Análisis Y Aplicaciones. Tesis Doctoral

García, M. (2002). Logística inversa, un nuevo coste a tener en cuenta por las empresas que operan en la Unión Europea (UE), Química e industria,(533), p. 30-34.

Bañegil, T. M. \& Rubio, S. (2003). Sistemas de logística inversa en la empresa.

Grupo de gestión de empresas. Universidad de Extremadura.

Cespón, R. \& Auxiliadora, M. (2003). Administración de la cadena de suministros. Manual para estudiantes de la especialidad de Ingeniería Industrial. Universidad Tecnológica Centroamericana de Honduras: UNITEC.

Miranda, F A. (1987). Organización y Planificación de la Producción. Tomo II. Ciudad Habana. Editorial ENPES. 
Tabla 4. Clasificación de los artículos teóricos (continuación)

García, A.T. (2008). Aplicación de un procedimiento para el diseño de la cadena de suministro de los residuos en la empresa Mármoles Centro del municipio de Fomento. Provincia Santi Spiritus.

Knudsen, J. (2005). Diseño y gestión de la cadena de suministro de los residuos agroindustriales de la caña de azúcar. Aplicación a los residuos agrícolas cañeros, el bagazo y las mieles. Tesis Doctoral.

Rubio, L. S. El sistema de logística inversa en la empresa: Análisis y Aplicaciones. Tesis Doctoral. Universidad de Extremadura.

Thierry, M. C., Salomon, M., Van Nunen, J. Y Van Wassenhove, L. (1995). Strategic issues in product recover y management. California Management Review 37 (2), 114-135.

Rubio, S. (2008). Retos medioambientales en las empresas: Logística inversa para la gestión de productos fuera de uso. Cursos de Verano Internacionales de la Universidad de Extremadura. Badajoz. Recuperado de http://educamarketing. unex.es/asignaturas/cursodeverano/Logisticalnversa_SergioRubio.pdf.

Díaz, A., Álvarez, M.J., González, P. (2004). Logística Inversa y Medio Ambiente: aspectos estratégicos y operativos. Madrid:, McGraw Hill, cop.

Elias, X. (2009). Reciclaje de residuos industriales: residuos sólidos urbanos y fangos de depuradora. Madrid, Díaz de Santos, cop. 2da. Ed.

Flkapper, D. P., et al. (2005). (ed.). Managing Closed-loop Suply Chains. Springer Berlin-Heidelberg, Alemania.

Fuente. Elaboración propia del autor. 


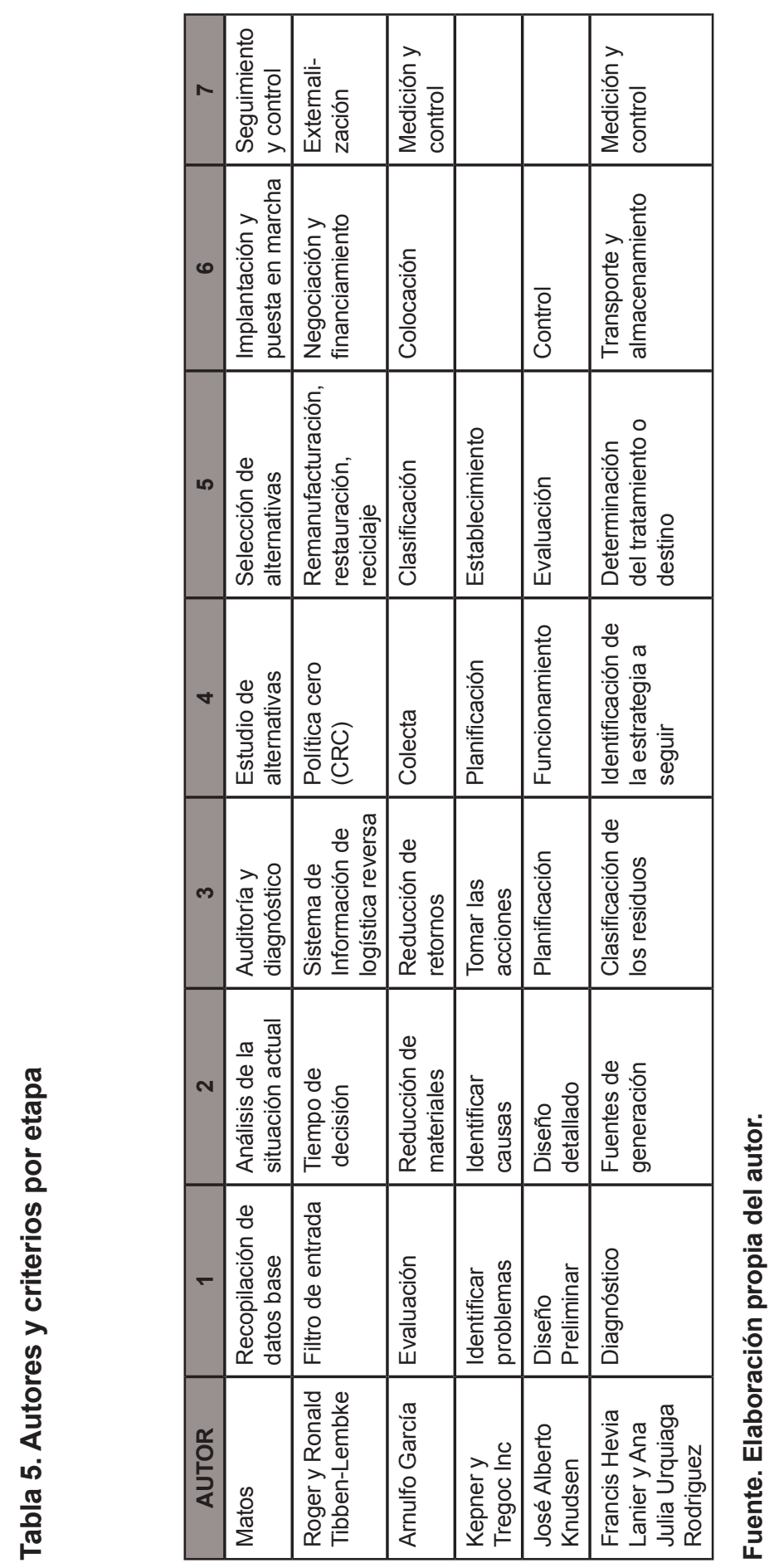




\subsection{Niveles de los diferentes actores en la logística inversa}

En la logística inversa participan actores con diferentes funciones, responsabilidades y niveles estratégicos que permiten lograr sus objetivos, alcanzar los beneficios potenciales y ejecutar los diversos procesos involucrados al mínimo costo y con niveles adecuados de desempeño.

Son responsables de los productos o materiales; en otras ocasiones son actores relacionados, especializados o clientes. Por ello, es importante que se identifique su rol, sus responsabilidades y los procesos de la logística inversa a participar y ejecutar para evitar problemas legales, operaciones y sociales.

- Actores principales: dentro de los cuales, se consideran los proveedores, distribuidores, minoristas, cliente y la empresa responsable de la recuperación del producto o productor.

- Actores especializados: los cuales ejecutan los procesos específicos de la logística inversa tales como: prestadores de servicio de transporte, almacenamiento, recicladores, operadores de reprocesamiento 0 eliminación de desechos.

- Actores relacionados: los cuales son organizaciones gubernamentales, ONG ambientalistas, entre otras, que afectan a la logística inversa de la cadena de suministros.

Estas actividades llevan asociadas políticas y decisiones a largo plazo de dirección, organización, planeación, programación, administración, coordinación y control de los sistemas y flujos interactuantes.

Se revisaron los artículos de tipo teórico con el objetivo de identificar estrategias logística inversa, encontrando 21 publicaciones que hablan específicamente de estrategias, las cuales tienen su origen en la logística comercial o empresarial. En los demás artículos que no mencionan específicamente estrategias. 


\section{Gestión de los residuos}

$\mathrm{E}$ I tratamiento tradicional de los residuos, no tenía en cuenta o no se preocupaba por la incidencia de los contaminantes. En la actualidad, existe una gran preocupación para el medio ambiente, con lo cual este tratamiento tradicional ha pasado a un modelo productivo y social limpio, en el que se minimiza la producción de residuos, bien disminuyendo su cuantía en origen el o bien mediante la aplicación de sistemas de tratamiento y recuperación. Todo esto se puede resumir en el principio de coeficiencia: Producir más limpio es más rentable que limpiar. En la gestión de los residuos también deben contemplarse las técnicas de eliminación, los tratamientos y el almacenamiento de los residuos especiales en lugares adecuados y seguros.

Thierry et al $(1995,114)$ define el concepto de gestión de productos recuperados como "la gestión de todos los productos, componentes y materiales usados y desechados por los consumidores, sobre los que el fabricante tiene cualquier tipo de responsabilidad y cuyo objetivo es recuperar tanto valor económico (y ecológico) como sea posible, reduciendo de esta forma la cantidad final de residuos". Estos autores señalan cinco opciones que puede utilizar la empresa para obtener un valor económico a partir de los residuos:

- Reparación: su objetivo es volver a poner al producto usado en condiciones de funcionamiento aunque, por lo general, la calidad de estos productos reparados suele ser inferior a la de los productos nuevos, como en el caso de los electrodomésticos, productos eléctricos y electrónicos.

- Restauración: supone devolver al producto usado unos niveles específicos de calidad (generalmente inferiores a los de los productos originales) y ampliar así su vida útil. Ejemplos de esta opción de recuperación se puede encontrar en la industria de la aviación civil y militar. 
- Refabricación: esta opción proporciona al producto usado unos estándares de calidad tan rigurosos como los de los productos originales consiguiendo unos costes de fabricación inferiores. Ejemplos de esta opción se encuentran en fotocopiadoras, electrodomésticos o en cámaras fotográficas desechables.

- Canibalismo: únicamente se recupera una pequeña parte de los componentes reutilizables los cuales se destinarán a las opciones de reparación, restauración y refabricación por ejemplo: componentes electrónicos, circuitos integrados, metales preciosos, etc.

- Reciclaje: consiste en recuperar el material con el que está fabricado el producto fuera de uso, para utilizarlo en la fabricación de nuevos productos. Es la opción más conocida y la de mayor aplicación por ejemplo: vidrio, papel, cartón, latas, etc.

Por su parte (Fleischmann et al, 1997), realizan una clasificación de acuerdo con el grado de descomposición que sufre el producto en el proceso de recuperación, considerando como opciones de gestión para la recuperación económica la reutilización de los productos, la reparación, la re fabricación y el reciclaje.

Las opciones existentes para la gestión de los residuos deben tener como principal objetivo su recuperación económica, por lo que dichas opciones deben satisfacer ciertas premisas:

- Que se trate realmente de un producto fuera de uso, es decir, que no satisfaga ya las necesidades del consumidor y este lo deseche.

- Que incorporen un valor añadido susceptible de ser recuperado a través de la cadena de suministro.

- Que se obtenga un nuevo ciclo de vida para el producto o para alguno de sus componentes.

De acuerdo con estas premisas, quizá fuera más adecuado no incluir la reparación de productos como una opción en la gestión de los residuos, en primer lugar, porque la recuperación del residuo se basa en la idea del 
aprovechamiento del valor que incorporan dichos productos y la actividad de reparación no recupera sino que sustituye.

La tabla 6 se identifica en artículos sobre las opciones de valor económico para los residuos.

\section{Tabla 6. Gestión de residuos}

\begin{tabular}{|c|c|}
\hline AUTOR & OPCIONES \\
\hline $\begin{array}{l}\text { Cespón, R., (1994). Cuba: Sistema para la proyección Tecnológica } \\
\text { de Instalaciones productivas de tratamiento térmico en fases } \\
\text { primarias inversionistas en Cuba. Tesis Doctoral. }\end{array}$ & \multirow{3}{*}{ Reparación } \\
\hline $\begin{array}{l}\text { González, H. C,s; Hernández Mi., R., y Cespón, R. (1998). Logística } \\
\text { de la distribución comercial, un enfoque sistémico. Revista Logística } \\
\text { Aplicada. (4) 28-33. (Ciudad de la Habana) }\end{array}$ & \\
\hline $\begin{array}{l}\text { Tibben-Lembke, R. Life after death (2002). Reverse logistics and } \\
\text { the product lifecycle. International Journal of Physical Distribution \& } \\
\text { Logistics Management. (3). }\end{array}$ & \\
\hline $\begin{array}{l}\text { Bridgewater, P. (1992). Fortalecimiento de áreas protegidas. En: } \\
\text { estrategia global para la Biodiversidad. UICN-WRI-PNUMA. p. 117- } \\
132\end{array}$ & \multirow{3}{*}{ Restauración } \\
\hline $\begin{array}{l}\text { Finegan, B. 1993. Procesos dinámicos en bosques naturales } \\
\text { tropicales. Curso de bases Ecológicas para la producción } \\
\text { sostenible. CATIE, Turrialba, Costa Rica. } 25 \mathrm{p} \text {. }\end{array}$ & \\
\hline $\begin{array}{l}\text { Moreno, C. (2003). Estrategia. Material de consulta para estudiantes } \\
\text { de RR. HH. Recuperado de: www.uch.edu.ar/rrhh }\end{array}$ & \\
\hline $\begin{array}{l}\text { Guide, V.D.R. Jr, Jayaraman, V., Srivastava, R. and Benton, W.C. } \\
\text { (2000). Supply-chain management for recoverable manufacturing } \\
\text { systems, Interfaces, (3). }\end{array}$ & Refabricación \\
\hline $\begin{array}{l}\text { Knudsen, J. (2005). Diseño y gestión de la cadena de suministro de } \\
\text { los residuos agroindustriales de la caña de azúcar. Tesis Doctoral. }\end{array}$ & Canibalismo \\
\hline $\begin{array}{l}\text { Gómez, D. (1998). Evaluación de impacto ambiental. Editorial } \\
\text { Agrícola Española, S. A. Madrid España. 63-100. }\end{array}$ & \multirow{3}{*}{ Reciclaje } \\
\hline $\begin{array}{l}\text { Fernández, A. (2004). Logística Inversa y Medio Ambiente. } \\
\text { Aspectos estratégicos y Operativo. España, Mc Graw Hill. }\end{array}$ & \\
\hline $\begin{array}{l}\text { Hernández, H. C. Milián, R. y Cespón, R. (1998). Logística de la } \\
\text { distribución comercial, un enfoque sistémico. Revista Logística } \\
\text { Aplicada. (4). 28-33. }\end{array}$ & \\
\hline
\end{tabular}

\section{Fuente. Elaboración propia del autor.}




\section{Conclusiones}

Se puede ver en las publicaciones que en los países más desarrollados como China, Japón, Europa y Estados Unidos hay más publicaciones, porque la legislación es más severa e involucra al productor, distribuidor y consumidor.

Debido a la conciencia mundial de proteger el medio ambiente y en especial la capa de ozono, se están incrementando los trabajos de investigación de la logística inversa en todos los sectores industriales.

El trámite efectivo del flujo físico de las mercancías que perdieron su vida útil y/o productos en proceso y materiales desechados e información relacionada desde el posconsumo, consumidor final, distribuidor y fabricación, apoyándose en la recolección y transporte hasta la opción de recuperación en reducción en la fuente, reutilización, refabricación, reciclaje y/o disposición final para recobrar su valor parcial o total y disminuir el impacto ambiental.

La literatura, conceptos, características, sirven como guía para realizar el proyecto de sistema de gestión de la logística inversa en el sector de la refrigeración en la ciudad de Bogotá. 


\section{Referencias bibliográficas}

Altay, N., y Green III, W. G. (2006). OR/MS research in disaster operations management. European Journal of Operational Research. 175(1), 475-493.

ALV Asociación de logística. (2008). ALV Asociación de logística. (s.e.)

Angulo, J. (2003). Logística Inversa. Recuperado from http://www.todologistica.com/online /site/58692/1.php.

Bañegil , T., y Rivero, P. (1998). ¿Cómo de verde es su marketing? EsicMarket 99.

Bereciartúa, y Echazarra. (2007). Logística Inversa. (s.e.)

Brito, y Daker. (2003). Aframework for Revere Logistics, in: Dekker, R. et al. Reverse logistics: Quantitative Models for Closed. Loop Supply Chapter1.

Carter, C., y Ellram, L. (1998). Reverse logistics: A review of the literature and framework for future investigation. Journal of Business Logistics 19 (1), 85-104.

Cespón Castro, R. (1994). Sistema para la proyección Tecnológica de Instalaciones productivas de tratamiento térmico en fases primarias inversionistas en Cuba. Tesis doctoral. (s.e.)

Council, R. L. (2007). Logística Inversa. (s.e.)

Fernández, y Gómez. (2005). Logística Inversa. (s.e.)

Fleischmann et al. (1997). Clasificación de acuerdo con el grado de descomposición que sufre el producto en el proceso de recuperación. (s.e.) 
Gaytan, J. (2012 ). Logística inversa una segunda oportunidad de negocio. International Logistics Summit y expo, Mexico D. F. Recuperado de: www.enfasis.com/Presentaciones/LS/2012/Talleres/Gaytan.pdf

Gómez Vieites, Á. (2007). Sistemas de información. Herramientas prácticas para la gestión empresarial. México: Alfa omega/Rama.

González, H., Hernández Milián, R., y Cespón Castro, R. (1998). Logística de la distribución comercial, un enfoque sistémico. Revista Logística Aplicada. 28-33.

Guide, J., y V. D. R. (2000). Production planning and control for remanufacturing industry practice and reseach needs. Journal of Operations Management 18. 467-483.

Hawks, K. (2006). Logística . (s.e.)

Hernández, R., Fernández , C., y Batista , P. (2006). Metodología de la investigación. McGraw-Hill.

Knemeyer, Ponzurick, y Logar. (2002). Logística Inversa.(s.e.)

Knudsen González, J. (2005). Diseño y gestión de la cadena de suministro de los residuos agroindustriales de la caña de azúcar. Aplicación a los residuos agrícolas cañeros el bagazo y las mieles. Tesis Doctoral. (s.e.)

Kotler, P. (1994). Marketing management: Analysis, planning, implementation, and control. Prentice Hall.

Krikke, Blanc, L., y Velde, V. d. (2003). Logística Inversa.

Monroy, N., Ahumada, M., y Cl. (2006). Reverse Logistics: Challenges for Industrial Engineering 23 Rev.ing. 23-33. Recuperado de: www.scielo. unal.edu.co/scielo.php.

Ortega, M. (2003). Logística Inversa. Situación actual de dos sectores significativos. V Congreso de Ingeniería de Organización Valladolid España. 
PNUMA. Programa de las Naciones Unidas para el Medio Ambiente División de Tecnología (2005). Manual de Medio Ambiente y Comercio: Programa de las Naciones Unidas para el Medio Ambiente, Instituto Internacional para el Desarrollo Sostenible. (2a ed.).

Ravi y Shankar. (2004). Logística Inversa. (s.e.)

Rogers, D., y Tibben-Lembke, R. S. (1999). Going backwards: Reverse Logistics Trends and practices. Reverse Logistics Executive Council. Nevada, USA. (s.e.)

Rojas, A., Salazar, S., Sepúlveda, A., et. al. (2006). Residuos sólidos domiciliarios: Logística: una herramienta moderna para afrontar este antiguo problema. Revista Ingeniería Industrial, 5 (2).Chile: Universidad del Bio-Bio. 77_87

Rubio, E. (2007). Logística Inversa. (s.e.)

Rubio, S. (2003). Sistemas de Logística Inversa en la empresa: análisis y aplicaciones. Tesis Doctoral Universidad de Extremadura. Recuperado el 15 de enero de 2011. Disponible en: http://dialnet.unirioja. es/servlet/dcfichero_tesis.

Rubio, S. (2008). Retos medioambientales en las empresas: logística inversa para la gestión de productos fuera de uso. Cursos de verano internacionales de la Universidad de Extremadura, Badajoz. Recuperado de: http://educamarketing.unex.es/asignaturas/cursodeverano/Logisticaln-versa_SergioRubio.pdf.

Sánchez, G. (2002). Desarrollo y medio ambiente: una mirada a Colombia. Economía y Desarrollo (1). (s.e.)

Thierry et al. (1995). Gestión de productos recuperados. 114. (s.e.)

Tibben-Lembke, R. (2002). Life after death: reverse logistics and the product lifecycle. International Journal of Physical Distribution y Logistics Management. 32(3). (s.e.) 
